\title{
1 Distinct functional roles of primate grasping hands and feet during arboreal quadrupedal \\ 2 locomotion
}

4 Biren A. Patel ${ }^{1, \dagger, \star}$, Ian J. Wallace ${ }^{2, \dagger}$, Doug M. Boyer ${ }^{3}$, Michael C. Granatosky ${ }^{3}$, Susan G.

5 Larson ${ }^{4}$, Jack T. Stern, Jr. ${ }^{4}$

6

$7 \quad{ }^{1}$ Department of Cell and Neurobiology, Keck School of Medicine, University of Southern

8 California, Los Angeles, CA 90033, USA

$9 \quad{ }^{2}$ Department of Human Evolutionary Biology, Harvard University, Cambridge, MA 02138,

10 USA

$11 \quad{ }^{3}$ Department of Evolutionary Anthropology, Duke University, Durham, NC 27708, USA

$12{ }^{4}$ Department of Anatomical Sciences, Stony Brook University Medical Center, Stony Brook, NY 1311794, USA

14

15 *correspondence: Biren A. Patel (birenpat@usc.edu)

$16{ }^{\dagger}$ Contributed equally to this work.

18 Abstract

19 It has long been thought that quadrupedal primates successfully occupy arboreal environments,

20 in part, by relying on their grasping feet to control balance and propulsion, which frees their

21 hands to test unstable branches and forage. If this interlimb decoupling of function is real, there

22 should be discernible differences in forelimb versus hind limb musculoskeletal control,

23 specifically in how manual and pedal digital flexor muscles are recruited to grasp during arboreal 
24 locomotion. New electromyography data from extrinsic flexor muscles in red ruffed lemurs

25 (Varecia rubra) walking on a simulated arboreal substrate reveal that toe flexors are activated at

26 relatively higher levels and for longer durations than finger flexors during stance phase. This

27 demonstrates that the extremities of primates indeed have different functional roles during

28 arboreal locomotion, with the feet emphasizing maintenance of secure grips. When this

29 dichotomous muscle activity pattern between the forelimbs and hind limbs is coupled with other

30 features of primate quadrupedal locomotion, including greater hind limb weight support and the

31 use of diagonal-sequence footfall patterns, a complex suite of biomechanical characters emerges

32 in primates that allow for the co-option of hands toward non-locomotor roles. Early selection for

33 limb functional differentiation in primates probably aided the evolution of fine manipulation

34 capabilities in the hands of bipedal humans.

\section{Keywords}

37 grasping hands, grasping feet, digital flexors, electromyography, Varecia, arboreal locomotion

\section{Introduction}

40 The early 20th century anatomist Wood Jones (1916) hypothesized that in ground-dwelling

41 quadrupedal animals, the forelimbs and hind limbs are equally responsible for controlling

42 balance and propulsion during locomotion, whereas in arboreal quadrupedal primates, these

43 functions are accomplished mainly by the hind limbs and their grasping feet. He referred to this

44 as the "emancipation of the forelimbs" in primates. Cartmill (1972) later expounded that by

45 relying on their hind limbs and grasping feet for support and propulsive forces, in particular the

46 big toe (hallux), primates free their hands for other critical tasks of arboreal living such as 
47 reaching out for and testing unstable branches, and facilitating foraging on thin terminal

48 branches. Wood Jones, Cartmill, and others have argued that this functional differentiation

49 between the forelimbs and hind limbs likely appeared early in primate evolution and served as

50 the basis for the eventual evolution of more specialized locomotor behaviors seen in living

51 primate taxa including vertical leaping in strepsirrhines, forelimb suspension in hominoids, and

52 bipedalism in humans (Wood Jones, 1916; Napier, 1967; Cartmill, 1972; Rollinson and Martin,

53 1981; Martin, 1990; Schmitt, 2003; Cartmill et al., 2007).

55 primate quadrupedalism and, in particular, for the distinct functional roles that the forelimbs and

56 hind limbs play during arboreal locomotion (Larson, 1998; Schmitt, 2003). During above-

57 branch pronograde locomotion, primates typically support more of their body mass on their hind

58 limbs (Kimura et al., 1979; Demes et al., 1994; Schmitt and Hanna, 2004; Franz et al., 2005;

59 Carlson and Demes, 2010), while in non-primate quadrupeds, body mass is supported more

60 uniformly across the limbs or more on the forelimbs than the hind limbs (Kimura et al., 1979;

61 Demes et al., 1994). Additionally, primate quadrupeds are highly unusual in their habitual use of

62 diagonal-sequence symmetrical gaits, in which each foot touchdown is followed by the contact

63 of the diagonally opposite hand (Muybridge, 1887; Hildebrand, 1967; Rollinson and Martin,

64 1981; Larson, 1998; Cartmill et al., 2002, 2007; Shapiro et al., 2014). This may be due, in part,

65 to an increase in the duration of the contact time of the foot relative to the hand (Cartmill et al.,

66 2002). In an arboreal environment, this gait pattern can potentially ensure that a foot is placed in

67 a protracted position under the animal's center of mass on a tested branch at the moment when

68 the contralateral hand strikes down on an untested substrate, while at the same time the

69 contralateral foot continues to maintain a tight grip for increased stability (Cartmill et al., 2002, 

82 (Tuttle, 1972).

2007; Fig. 1).

Electrophysiological experiments of the primary sensorimotor cortex in pronograde monkeys have shown that the point-to-point mirroring of shape and function (i.e., somatotopic representation) of the five toes is fused, and thus all digits likely function as a unit during arboreal locomotion (Hashimoto et al., 2013). In contrast, neural mapping of the fingers shows that the five digits are independent of each other, and they are therefore better suited for finer dexterity and manipulation than the toes. Complementing this, the gross morphology of various non-human primates reveals that the tendons of extrinsic digital flexor muscles of the hands have independent insertions and likewise control of different distal joints, whereas the homologous tendons in the foot often fuse together before inserting and thereby act on the same joints to produce motion (Hartman and Straus, 1971; Lewis, 1989). Additionally, relative weights of total leg and foot muscles are generally greater than total forearm and hand muscles in many primates

Despite the evidence suggesting limb functional differentiation during locomotion, coupled with somatosensory and gross morphological differences between the hand and foot in primates, a critical issue remains open to question: are the muscles responsible for grasping recruited differently during arboreal quadrupedal locomotion? Specifically, do the hind limb digital flexor muscles exhibit higher, more regular, stride-correlated activity during stance than their forelimb counterparts? Rhesus macaques walking on treadmills exhibit some difference in the recruitment patterns of their digital flexor muscles of the forelimb versus hind limb (Courtine et al., 2005); however, treadmill walking cannot provide sufficient insight on the role these muscles play in grasping. For unrestrained arboreal movements, this possibility is only supported by indirect experimental evidence. For example, in common marmosets locomoting on 
93 simulated arboreal supports, hind limb stabilizing muscular torques, estimated from kinematics

94 and force transducer data, are higher than forelimb muscular torques, indicating that the former

95 play a greater role in maintaining lateral stability on branches (Chadwell and Young, 2015). In

96 addition, dynamic palmar/plantar pressure studies of Japanese macaques walking on simulated

97 branches show that peak pressures between digits 1 and 2 of the foot are significantly higher

98 compared to the homologous digits in the hand, thus suggesting that monkeys grasp with their

99 hallux using higher forces than with their pollex (thumb) (Higurashi et al., 2010). The fact that

100 intrinsic muscles controlling the hallux in adduction and flexion are relatively greater in size than

101 those that move the pollex offers additional support for this interpretation (Tuttle, 1972).

102

In the present study, we investigate this issue further by analyzing the activity patterns of

103 extrinsic toe and finger flexor muscles using telemetered electromyography (EMG) in red ruffed

104 lemurs (Varecia rubra) during movement on a simulated arboreal substrate. Ruffed lemurs are an

105 ideal model species because like nearly all extant arboreal primate quadrupeds, they habitually

106 use diagonal-sequence gaits (Cartmill et al., 2002; see also supplemental online material (SOM)

107 Figure S1) and support most of their body mass on their hind limbs (see SOM Table S1).

108 Additionally, studies have demonstrates that Varecia primarily uses tiny $(<2.5 \mathrm{~cm})$ and small

109 (2.5-10 cm) branches during locomotion and while foraging (Dagosto, 1994, 1995). This habitat

110 use closely matches the terminal branch environment in which the earliest primates were thought

111 to have evolved. Moreover, evaluation of grasping in this model is relevant for understanding

112 the primitive grasping mechanisms of primates, and possibly their evolution, since ruffed lemurs

113 are more similar to Eocene euprimates (e.g., adapids) than to anthropoids (e.g., macaques) in the

114 structure of their hand and foot grasping complexes, including features related to phalangeal and

115 metapodial proportions, relative pollex and hallux lengths, degree of pollical divergence, and 
116 hallucal grasp types (Gebo, 1985; Hamrick and Alexander, 1996; Kirk et al., 2008; Maiolino et

117 al., 2012; Patel et al., 2012b; Boyer et al., 2013).

\section{Materials and methods}

120 All experimental and analytical procedures have been detailed extensively elsewhere (Boyer et 121 al., 2007) and are only summarized here. All procedures were approved by the IACUC of Stony 122 Brook University and Duke University.

123 The subjects were three adult female red ruffed lemurs (Varecia rubra). EMG recordings

124 were obtained from the extrinsic finger flexors [flexor digitorum superficialis (FDS), flexor

125 digitorum profundus (FDP)] and the extrinsic toe flexors [flexor digitorum tibialis (FDT), flexor

126 digitorum fibularis (FDF)]. Although the intrinsic flexors muscles of the hand and foot

127 contribute to overall forces applied by the digits during grasping (Gyambibi and Lemelin, 2013),

128 we focused our study on only the extrinsic muscles rather than intrinsic flexors because the latter

129 are more invasive to sample owing to the fact that electrodes would need to be inserted through

130 the palmar and plantar surfaces and doing so would likely disrupt how the animals use their

131 hands and feet during locomotion. Bipolar fine-wire electrodes $(50 \mu \mathrm{m})$ were threaded through a

132 hypodermic needle (25 gauge) and inserted into the middle of muscle bellies while the subject

133 was under anesthesia. Each muscle was back-stimulated with a sinusoidal current (200-500 $\mu$ A;

$13450 \mathrm{~Hz}$ ) to ensure proper electrode placement and verify action of muscles in isolation. Electrodes

135 were connected to a 112 g, 4-channel FM telemetry transmitter (Bio-Sentry Telemetry, Torrance,

136 CA, USA) attached to a non-restrictive harness worn by the subject. Subjects were placed in a

137 large enclosure made of chain-link fence that contained a horizontally positioned pole (length: 4

$138 \mathrm{~m}$; diameter $3.1 \mathrm{~cm}$ ) elevated $\sim 1.0 \mathrm{~m}$ above the floor to simulate an arboreal substrate. A branch 
139 diameter of $3.1 \mathrm{~cm}$ is considered to be relatively narrow for an animal the size of Varecia rubra

140 (with a species mean of 3.57 kg; see Goodenberger et al. [2015] and Stevens [2008]), but is

141 considered to be within the range of the most commonly utilized substrate diameters for this

142 species (Dagosto, 1994, 1995). Within the enclosure, subjects were allowed to move freely and

143 only strides using symmetrical gaits (Hildebrand, 1967) without any whole-body aerial phase

144 were studied.

145 EMG signals were detected by an FM receiver that sent demodulated EMG output to a

146 SCXI-1000 A-D converter (National Instruments, Austin, TX, USA), whose signal was acquired

147 at $2700 \mathrm{~Hz}$ with LabView software (National Instruments). Simultaneous video recorded at 60

$148 \mathrm{~Hz}$ was superimposed onto digital EMG recordings to determine the relationship between subject

149 behavior and muscle activity. Videos enabled identification of full step cycles and kinematic

150 events including limb touchdown and liftoff. A custom Fortran program read raw EMG data

151 files, identified data points corresponding to touchdown and liftoff, and calculated the root mean

152 square (r.m.s.) of EMG for all samples of stance and swing phase at 1.85 ms intervals using a

153 time constant of 41.85 ms. The r.m.s. EMG values were scaled as a percentage of the maximum

154 r.m.s. EMG observed for each muscle in each experiment, which typically occurred during fence

155 climbing.

156 Muscle activity was analyzed in two ways. First, another Fortran program read sample

157 files containing r.m.s. EMG information, equalized them with regard to phase duration, and

158 calculated at 1\% intervals the weighted average distribution of the r.m.s. EMG over a step cycle

159 for all experiments. From these data, the average activity profiles for each muscle over an entire

160 step cycle were created to qualitatively compare activity patterns across muscles. Second, the

161 duration and peak r.m.s. EMG values for each muscle during stance phase of each analyzed 
162 stride were identified (see SOM for raw data). A mixed-model full factorial designed analysis

163 was performed (to account for pseudo-replication; e.g., Patel et al., 2015) with muscle as the

164 fixed factor and animal subject as the random factor (with experiment date nested within), and

165 this was followed by post-hoc analyses performed using Tukey’s honest significant difference

166 (HSD) tests (see also SOM). All statistical analyses were done in JMP Pro v. 10.0 software

167 (SAS Institute, Inc., Cary, NC, USA) with alpha levels set at 0.001.

168

169 Results

170 A total of four recording sessions for the three animal subjects resulted in 80 analyzable strides

171 for each muscle, except FDT for which 69 strides could be evaluated (Table 1). Datasets

172 supporting this article have been uploaded as part of the SOM. All muscles analyzed were active

173 during stance phase but not swing phase, with the highest levels of activity occurring shortly

174 after limb touchdown (Fig. 2). Mixed-model ANOVAs revealed that both duration $\left(\mathrm{F}_{[3,303]}=\right.$

175 150.99, $p<0.001)$ and intensity of muscle activity $\left(\mathrm{F}_{[3,303]}=50.25, p<0.001\right)$ differed

176 significantly between muscles. Furthermore, post-hoc analyses showed that all muscles differed

177 significantly from each other ( $p<0.001$ for each comparison) and that the toe flexors (FDF,

178 FDT) were active for significantly longer durations and with relatively higher magnitudes than

179 the finger flexors (FDS, FDP) (Table 2; Fig. 3). In the foot, FDF and FDT were activated for, on

180 average ( \pm 1 standard deviation), $89.25 \pm 7.8 \%$ and $65.91 \pm 17.3 \%$ of the duration of stance

181 phase, respectively. In the hand, durations were lower with FDS and FDP activated for only

$18253.93 \pm 28.9 \%$ and $28.15 \pm 17.5 \%$ of stance, respectively. For muscle activation intensity, in the

183 foot, peak activity of FDF and FDT reached $35.21 \pm 12.5 \%$ and $28.07 \pm 15.2 \%$ of maximum 
184 burst, respectively. In the hand, peak activity of FDS and FDP reached $21.09 \pm 10.3 \%$ and 14.46

$185 \pm 6.5 \%$ of maximum burst, respectively.

186

187 Discussion

188 Most researchers agree that arboreality shaped the unusual characteristics of primate quadrupedal

189 locomotion (e.g., Wood Jones, 1916; Napier, 1967; Cartmill, 1972; Martin, 1990; Bloch and

190 Boyer, 2002; Schmitt, 2003; Cartmill et al., 2007). To occupy an exclusively arboreal habitat,

191 primates face significant locomotor and sensory challenges. They must navigate a complex

192 three-dimensional environment often composed of flexible substrates oriented in multiple

193 directions, and do so with sufficient stability to allow efficient and safe foraging. In part,

194 prehensile proportions of hands and feet help overcome these challenges. However,

195 observational data (Wood Jones, 1916; Napier, 1967; Cartmill, 1972) supported by indirect

196 empirical biomechanical data derived from analyses of gait, kinematics, kinetics, and hand/foot

197 pressure patterns (Muybridge, 1887; Hildebrand, 1967; Kimura et al., 1979; Rollinson and

198 Martin, 1981; Szalay and Dagosto, 1988; Demes et al., 1994; Larson, 1998; Cartmill et al., 2002;

199 Schmitt, 2003; Schmitt and Hanna, 2004; Franz et al., 2005; Cartmill et al., 2007; Carlson and

200 Demes, 2010; Higurashi et al., 2010; Shapiro et al., 2014; Chadwell and Young, 2015) continue

201 to suggest that it may be hind limb grasping with the feet that plays a more dominant role to

202 maintain stability in an arboreal milieu. In doing so, they would leave the grasping hands

203 available for more manipulative tasks. By investigating motor patterns (i.e., EMG) during

204 relevant dynamic activities (cf. Herrel et al., 2008) such as arboreal locomotion, it is possible to

205 identify whether pedal and manual grasping control differs. Our data on muscle recruitment

206 patterns assessed by telemetered EMG show that the toe flexors are activated at relatively higher 
207 levels and for longer durations than the finger flexors in red ruffed lemurs walking on simulated

208 arboreal branches. These findings imply less reliance on forelimb grasps during quadrupedal

209 locomotion, which creates the potential for directing the hands towards other roles (e.g.,

210 Georgopoulos and Grillner, 1989; Hashimoto et al., 2013; Toussaint et al., 2013).

211 Additional empirical data are needed from other primates and possibly other mammals

212 capable of grasping (e.g., some arboreal marsupials) to confirm the observed differences in

213 neuromuscular control across limb pairs. Nevertheless, previously reported muscle activity

214 patterns appear different in habitually terrestrial mammals (e.g., cats and dogs), which tend to

215 recruit their forelimb digital flexors with the same, if not more, duration and intensity as their

216 hind limb flexors during locomotion (Tokuriki, 1973; English, 1978; Rasmussen et al., 1978).

217 Therefore it remains possible that a greater emphasis on hind limb grasping is a primate-specific

218 phenomena, or at least is stereotypical for arboreal mammals with grasping appendages.

219 A growing body of literature shows that a difference in the recruitment of synergistic

220 muscles within limbs is common (e.g., O’Donovan et al., 1982; Ahn and Full, 2002; Higham et

221 al., 2008), and this includes the digital flexor muscles (i.e., FDS vs. FDP; FDF vs. FDT). In red

222 ruffed lemurs and baboons, FDS is active for a longer period of time and with relatively higher

223 magnitudes than FDP during stance phase above branches and on the ground (Patel et al., 2012a;

224 this study). In non-human primates, as well as in cats, FDF is more active than FDT (Goslow et

225 al., 1972; O’Donovan et al., 1982; Fleshman et al., 1984; Trank and Smith, 1996; Courtine et al.,

226 2005; Boyer et al., 2007; Patel et al., 2015; this study). The one known exception to this is in

227 slow lorises that use their FDT more than their FDF during powerful pedal grasping bouts

228 (Kingston et al., 2010). This difference may be related to the fact that FDT is the more massive 
229 of the two in slow lorises (Grand, 1977), whereas FDF is relatively larger in other primates

230 (Murie and Mivart, 1872). Irrespective of whether FDF (as in most primates) or FDT (as in slow

231 lorises) is more active, it is clear that one is more dominant in pedal grasping, and it is this

232 muscle that has a more significant role in flexing the hallux, a key adaptation for stability during

233 arboreal locomotion.

234 The findings presented here agree with previous studies describing the unusual features

235 of primate quadrupedal biomechanics (reviewed in Larson [1998] and Schmitt [2003]), and

236 provide further empirical evidence reinforcing the century-old hypothesis that key hind limb

237 adaptations of the primate neuromusculoskeletal system "emancipate” the forelimbs in their

238 selection potential (Wood Jones, 1916). In addition to generalized quadrupedal locomotion,

239 living primates use other specialized locomotor behaviors including forelimb suspension, hind

240 limb grasp-leaping, quadrumanus bridging, and bipedalism. Each form requires decoupled

241 neuromuscular control and differential use of manual and pedal grasping, or in the case of the

242 latter, a complete independence of the hands from locomotor duties. As proposed by Wood

243 Jones (1916) and his successors, the early neuromusculoskeletal evolution of functional limb

244 differentiation in primates may have allowed for these specialized forms of locomotion to 245 evolve.

246 Evidence of relatively long manual versus pedal phalanges and of an opposable hallux is

247 present in early Eocene euprimates (Kirk et al., 2008; Maiolino et al., 2012; Boyer et al., 2013).

248 These traits may have evolved as early as the late Paleocene in some closely related primate

249 ancestors (e.g., Carpolestes; Hamrick and Alexander, 1996; Bloch and Boyer, 2002). Primate

250 manual phalanges are typically relatively longer than their pedal counterparts (Jungers et al.,

251 2005), which enhances manual prehensility including the ability to manipulate objects such as 
252 food resources (Cartmill, 1972; Lemelin and Grafton, 1998). A relatively long and robust 253 opposable hallux, characteristic of all arboreal primates (Gebo, 1985; Lewis, 1989; Gebo, 2004;

254 Sargis et al., 2007), facilitates balance and stability during stance phase of an arboreal stride 255 when actively wrapped around a branch (Boyer et al., 2007; Kingston et al., 2010; Patel et al., 256 2015; this study). Given that the hands and feet in our extant lemur model are morphologically 257 similar to those of early euprimates of the Eocene (Hamrick and Alexander, 1996; Maiolino et 258 al., 2012; Boyer et al., 2013), the results presented here lend additional support to the hypothesis 259 (Martin, 1990; Larson, 1998) that the functional differentiation between the forelimbs and hind 260 limbs that distinguishes primate locomotor biomechanics, including differential use of the 261 forelimbs and hind limbs in grasping, likely occurred early in our evolutionary history.

263 Acknowledgements. Kristin Fuehrer-Lasek assisted with animal training. Luci Betti-Nash

264 created Figure 1. Matt Cartmill provided data for SOM Figure S1. Financial support was 265 provided by the National Science Foundation (grant \# BCS 0509190) and the Force and Motion 266 Foundation.

268 Author contributions. BAP and DMB designed the study; BAP, DMB, SGL, JTS performed 269 the experiments; IJW analyzed the EMG data; MCG provided essential supporting data; BAP 270 and IJW drafted the manuscript; all authors gave final approval for publication.

\section{References}

273 Ahn, A.N., Full, R.J., 2002. A motor and a brake: two leg extensor muscles acting at the same 274 joint manage energy differently in a running insect. J. Exp. Biol. 205, 379-389. 
276 Bloch, J.I., Boyer, D.M., 2002. Grasping primate origins. Science 298, 1606-1610.

278 Boyer, D.M., Patel B.A., Larson, S.G., Stern, J.T. Jr., 2007. Telemetered electromyography of 279 peroneus longus in Varecia variegata and Eulemur rubriventer: implications for the functional significance of a large peroneal process. J. Hum. Evol. 53, 119-134.

281

Boyer, D.M., Yapuncich, G.S., Chester, S.G.B., Bloch, J.I., Godinot, M., 2013. Hands of early primates. Yrbk. Phys. Anthropol. 57, 33-78. different substrates. Am. J. Phys. Anthropol. 142, 273-286.

Cartmill, M., 1972. Arboreal adaptations and the origin of the order Primates. In: Tuttle, R.H.

Carlson, K.J., Demes, B., 2010. Gait dynamics of Cebus apella during quadrupedalism on (Ed.), The Functional and Evolutionary Biology of Primates. Adline-Atherton, Chicago, pp. 97-122.

294

295 Cartmill, M., Lemelin, P., Schmitt, D., 2007. Primate gaits and primate origins. In: Ravosa, M.J., 296 Dagosto, M. (Eds), Primate Origins: Adaptations and Evolution. Springer, New York, pp. 403-435. 
299 Chadwell, B.A., Young, J.W., 2015. Angular momentum and arboreal stability in common marmosets (Callithrix jacchus). Am. J. Phys. Anthropol. 156, 565-576.

301

302 Courtine, C., Roy, R.R., Hodgson, J., McKay, H., Raven, J., Zhong, H., Tang, H., Tuszynski, M.H., Edgerton, V.R., 2005. Kinematic and EMG determinants in quadrupedal locomotion of a non-human primate (rhesus). J. Neurophysiol. 93, 3127-3145.

Dagosto, M., 1994. Testing positional behavior of Malagasy lemurs: a randomization approach. Am. J. Phys. Anthropol. 94, 189-202.

Dagosto, M., 1995. Seasonal variation in positional behavior of Malagasy lemurs. Int. J.

312 Demes, B., Larson, S.G., Stern, J.T. Jr., Jungers, W.L., Biknevicius, A.R., Schmitt, D., 1994. The kinetics of primate quadrupedalism: hindlimb drive reconsidered. J. Hum. Evol. 26, 353374. stepping in the cat. J. Exp. Biol. 76, 105-122. 
319 Fleshman, J.W., Lev-Tov, A., Burke, R.E., 1984. Peripheral and central control of flexor

320 digitorum longus and flexor hallucis longus motoneurons: the synaptic basis of functional 321 diversity. Exp. Brain Res. 54, 133-149.

322

323 Franz, T.M., Demes, B., Carlson, K.J., 2005. Gait mechanics of lemurid primates on terrestrial and arboreal substrates. J. Hum. Evol. 48, 199-217.

325

326

327

Gyambibi, A., Lemelin, P., 2013. Comparative and quantitative myology of the forearm and hand of prosimain primates. Anat. Rec. 296, 1196-1206.

328

329

Gebo, D.L., 1985. The nature of the primate grasping foot. Am. J. Phys. Anthropol. 67, 269-277.

330

331 Gebo, D.L., 2004. A shrew-sized origin for primates. Yrbk. Phys. Anthropol. 47, 40-62.

332

333 Georgopoulos, A.P., Grillner, S., 1989. Visuomotor coordination in reaching and locomotion.

$334 \quad$ Science 245, 1209-1210.

335

336 Goodenberger, K.E., Boyer, D.M., Orr, C.M., Jacobs, R.L., Femiani, J.C., Patel, B.A., 2015. Functional morphology of the hallucal metatarsal with implications for inferring grasping

339

340 Goslow, G.E. Jr., Stauffer, E.K., Nemeth, W.C., Stuart, D.G., 1972. Digit flexor muscles in the 341 cat: their action and motor units. J. Morphol. 137, 335-342. 
343 Grand, T.I., 1977. Body weight: its relation to tissue composition, segment distribution, and motor function. I. Interspecific Comparisons. Am. J. Phys. Anthropol. 47, 211-239.

Hamrick, M.W., Alexander, J.P., 1996. The hand skeleton of Notharctus tenebrosus (Primate, Notharctidae) and its significance for the origin of the primate hand. Am. Mus. Novitates

Hartman, C.G., Straus, W.L. Jr., 1971. The Anatomy of the Rhesus Monkey. Hafner, New York.

Hashimoto, T., Ueno, K., Ogawa, A., Asamizuya, T., Suzuki, C., Cheng, K., Tanaka, M., Taoka, M., Iwamura, Y., Suwa, G., Iriki, A., 2013. Hand before foot? Cortical somatotopy suggests manual dexterity is primitive and evolved independently of bipedalism. Phil. Trans. R. Soc. B. 368, 20120417.

Herrel, A., Schaerlaeken, V., Ross, C., Meyers, J., Nishikawa, K., Abdala, V., Manzano, A., Aerts, P., 2008. Electromyography and the evolution of motor control: limitations and

Higham, T.E., Biewener, A.A., Wakeling, J.M., 2008. Functional diversification within and between muscle synergists during locomotion. Biol. Lett. 4, 41-44. 
364 Higurashi, Y., Hirasaki, E., Kumakura, H., 2010. Palmar and plantar pressure while walking on a horizontal ladder and single pole in Macaca fuscata. Int. J. Primatol. 31, 181-190.

366

367

Hildebrand, M., 1967. Symmetrical gaits of primates. Am. J. Phys. Anthropol. 26, 119-130.

368

369

370

Jungers, W.L., Lemelin, P., Godfrey, L.R., Wunderlich, R.E., Burney, D.A., Simons, E.L., Chatrath, P.S., James, H.F., Randria, G.F., 2005. The hands and feet of Archaeolemur: metrical affinities and their functional significance. J. Hum. Evol. 49, 36-55.

372

373

Kimura, T., Okada, M., Ishida, H., 1979. Kinesiological characteristics of primate walking: its significance in human walking. In: Morbeck, M.E., Preuschoft, H., Gomberg, N. (Eds), Environment, Behavior and Morphology: Dynamic Interactions in Primates. G. Fischer, New York, pp. 297-311.

377

Kingston, A.K., Boyer, D.M., Patel, B.A., Larson, S.G., Stern, J.T. Jr., 2010. Hallucal grasping in Nycticebus coucang: further implications for the functional significance of a large peroneal process. J. Hum. Evol. 58, 33-42.

Kirk, E.C., Lemelin, P., Hamrick, M.W., Boyer, D.M., Bloch, J.I., 2008. Intrinsic hand proportions of euarchontans and other mammals: implications for the locomotor behavior of plesiadapiforms. J. Hum. Evol. 55, 278-299. 
386

387

388

389

390

391

392

393

394

395

396

397

398

399

400

401

402

403

404

405

406

407

408

Larson, S.G., 1998. Unique aspects of quadrupedal locomotion in non-human primates. In: Strasser, E., Fleagle, J., Rosenberger, A., McHenry, H. (Eds), Primate Locomotion: Recent Advances. Plenum Press, New York, pp. 157-173.

Lemelin, P., Graftron, B.W., 1998. Grasping performance in Saguinus midas and the evolution of hand prehensility in primates. In: Strasser, E., Fleagle, J., Rosenberger, A., McHenry, H. (Eds), Primate Locomotion: Recent Advances. Plenum Press, New York, pp. 131-144.

Lewis, O.J., 1989. Functional Morphology of the Evolving Hand and Foot. Clarendon, Oxford.

Maiolino, S., Boyer, D.M., Bloch, J.I., Gilbert, C.C., Groenke, J., 2012. Evidence for a grooming claw in a North American adapiform primate: implications for anthropoid origins. PLOS ONE 7, e29135.

Martin, R.D., 1990. Primate Origins and Evolution. Princeton University Press, Princeton.

Murie, J., Mivart, S.G., 1872. On the anatomy of the Lemuroidea. Trans. Zool. Soc. Lond. 7, 1113.

Muybridge, E., 1887. Animal Locomotion. Dover, New York.

Napier, J.R., 1967. Evolutionary aspects of primate locomotion. Am. J. Phys. Anthropol. 27, 333-342. 
410 O’Donovan, M.J., Pinter, M.J., Dum, R.P., Burke, R.E., 1982. Actions of FDL and FHL muscles in intact cats: functional dissociation between anatomical synergists. J. Neurophysiol. 47, 1126-1143

413

414 Patel, B.A., Larson, S.G., Stern, J.T. Jr., 2012a. Electromyography of wrist and finger flexor muscles in olive baboons (Papio anubis). J. Exp. Biol. 215, 115-123.

Patel, B.A., Seiffert, E.R., Boyer, D.M., Jacobs, R.L., St. Clair, E.M., Simons, E.L., 2012b. New primate first metatarsals from the Paleogene of Egypt and the origins of the anthropoid big toe. J. Hum. Evol. 63, 99-120.

420

Patel, B.A., Larson, S.G., Stern, J.T. Jr., 2015. Electromyography of crural and pedal muscles in tufted capuchin monkeys (Sapajus apella): implications for hallucal grasping behavior and first metatarsal morphology in euprimates. Am. J. Phys. Anthropol. 156, 553-564.

424

Rasmussen, S., Chan, A.K., Goslow, G.E. Jr., 1978. The cat step cycle: electromyographic patterns for hindlimb muscles during posture and unrestrained locomotion. J. Morph.

428 155, 253-270.

430

Rollinson, J., Martin, R.D., 1981. Comparative aspects of primate locomotion, with special reference to arboreal cercopithecines. Symp. Zool. Soc. Lond. 48, 377-427. 
432 Sargis, E.J., Boyer, D.M., Bloch, J.I., Silcox, M.T., 2007. Evolution of pedal grasping in

433 Primates. J. Hum. Evol. 53, 103-107.

434

435 Schmitt, D., 2003. Insights into the evolution of human bipedalism from experimental studies of 436 humans and other primates. J. Exp. Biol. 206, 1437-1448.

437

438 Schmitt, D., Hanna, J.B., 2004. Substrate alters forelimb to hindlimb peak force ratios in 439 primates. J. Hum. Evol. 46, 237-252.

440

441 Shapiro, L.J., Young, J.W., VandeBerg, J.L., 2014. Body size and the small branch niche using 442 marsupial ontogeny to model primate locomotor evolution. J. Hum. Evol. 68, 14-31.

443

444 Szalay, F.S., Dagosto, M., 1988. Evolution of hallucial grasping in the Primates. J. Hum. Evol.

445 $17,1-33$.

446

447 Tokuriki, M., 1973. Electromyographic and joint-mechanical studies in quadrupedal locomotion. I. Walk. Jap. J. Vet. Sci. 35, 433-446.

449

450 Toussaint, S., Reghem, E., Chotard, H., Herrel, A., Ross, C.F., Poydebat, E., 2013. Food acquisition on arboreal substrates by the grey mouse lemur: implication for primate grasping evolution. J. Zool. 291, 235-242.

453 
454 Tuttle, R., 1972. Relative mass of the cheiridial muscles in catarrhine primates. In: Tuttle, R.H.

455 (Ed), The Functional and Evolutionary Biology of Primates. Aldine-Atherton, Chicago,

$456 \quad$ pp. 262-291.

457

458 Trank, T.V., Smith, J.L., 1996. Adaptive control for backward quadrupedal walking VI.

459 Metatarsophalangeal joint dynamics and motor patterns of digit muscles. J. Neurophysiol.

$460 \quad 75,678-694$.

461

462 Wood Jones, F., 1916. Arboreal Man. Edward Arnold, London.

463 


\section{Captions}

465 Table 1. Number of steps sampled for toe flexors (FDF, FDT) and finger flexors (FDS, FDP) in 466 three subjects and across four experiments.

Table 2. Mean, least-squares (LS) mean, and 1 standard error of LS mean values of relative 469 muscle activity duration and peak activity intensity in toe flexors (FDF, FDT) and finger flexors

470 (FDS, FDP). All muscles are significantly different in relative duration and relative peak activity 471 (see also Fig. 3).

472

473 Figure 1. Red ruffed lemur walking on a simulated arboreal substrate and wearing a jumpsuit 474 and telemetry transmitter attached to a harness. The subject is using a diagonal-sequence gait in 475 which the right foot touchdown is followed by the contact of the left hand. Stability is 476 maintained by the grasping feet.

478 Figure 2. Muscle activity determined by EMG in finger flexors (FDS, FDP) and toe flexors 479 (FDF, FDT). (A) Representative raw EMG waves for two consecutive step cycles. Stance phase 480 begins with touchdown (TD) and ends with liftoff (LO). (B) Average distribution of r.m.s. EMG 481 over a step cycle for all experiments. EMG values have been scaled to a percentage of maximum 482 burst. Only r.m.s. EMG values greater than 5\% of maximum burst were considered meaningful 483 and only these are illustrated. 
485 Figure 3. Least squares (LS) means and 1 standard error for relative muscle duration (A) and 486 relative peak activity (B) in toe flexors (FDF, FDT) and finger flexors (FDS, FDP). All muscles 487 are significantly different in relative duration and relative peak activity (see also Table 2). 488 
fig 1

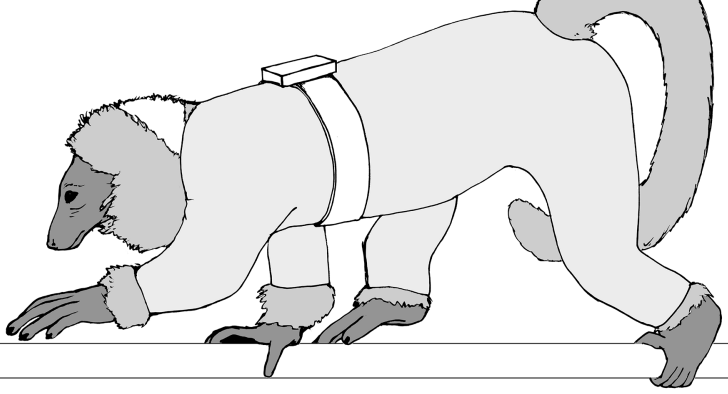




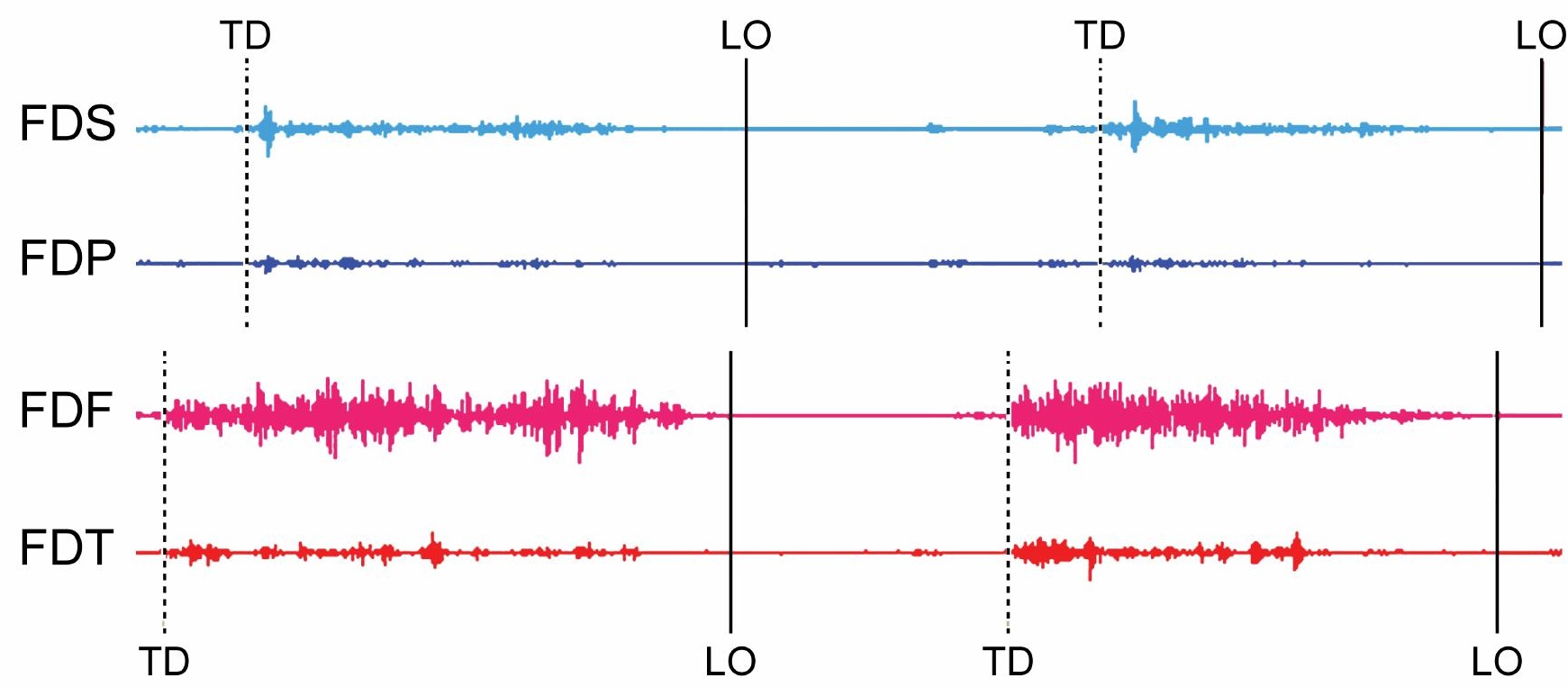

B
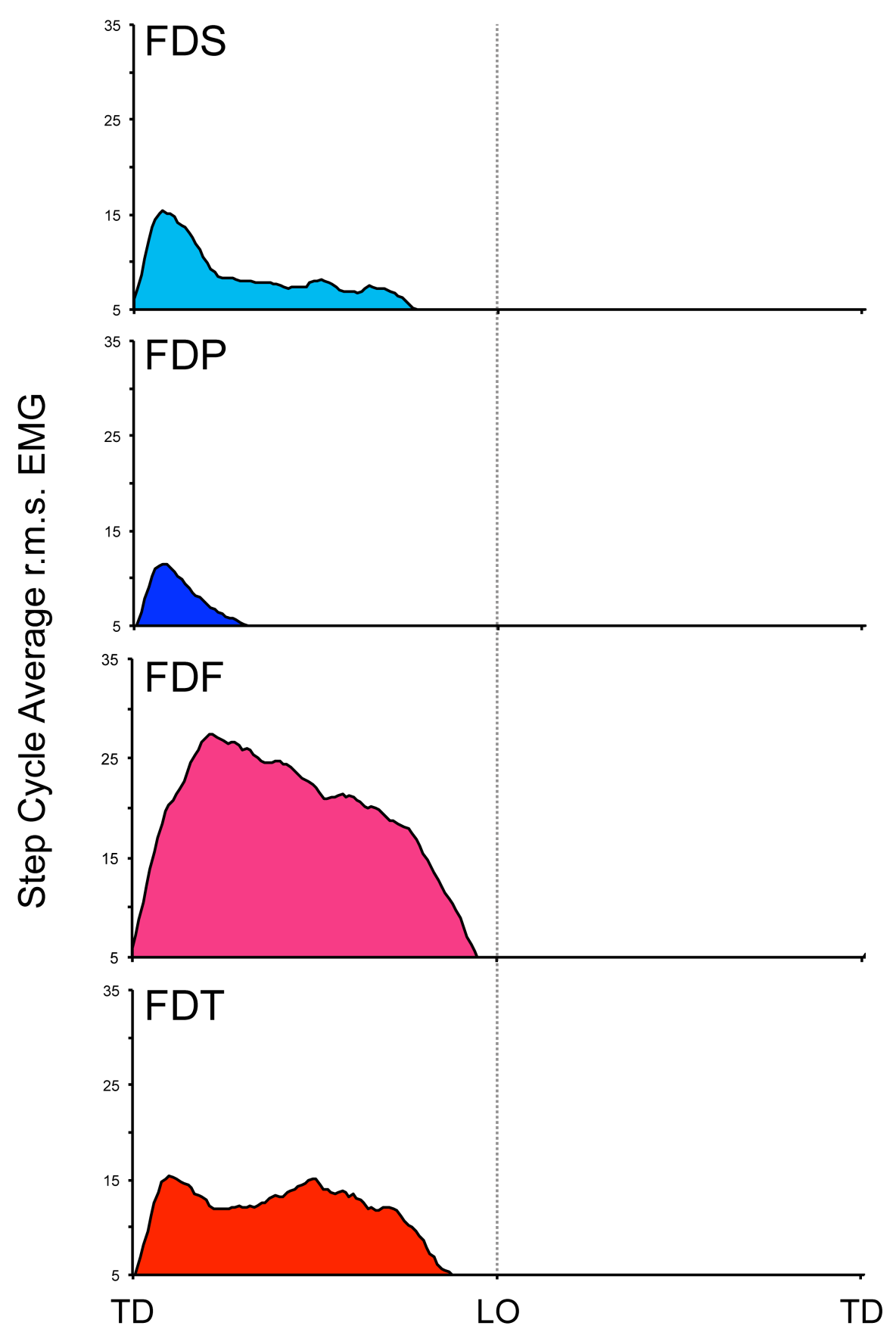


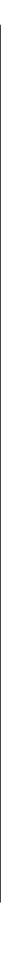

B

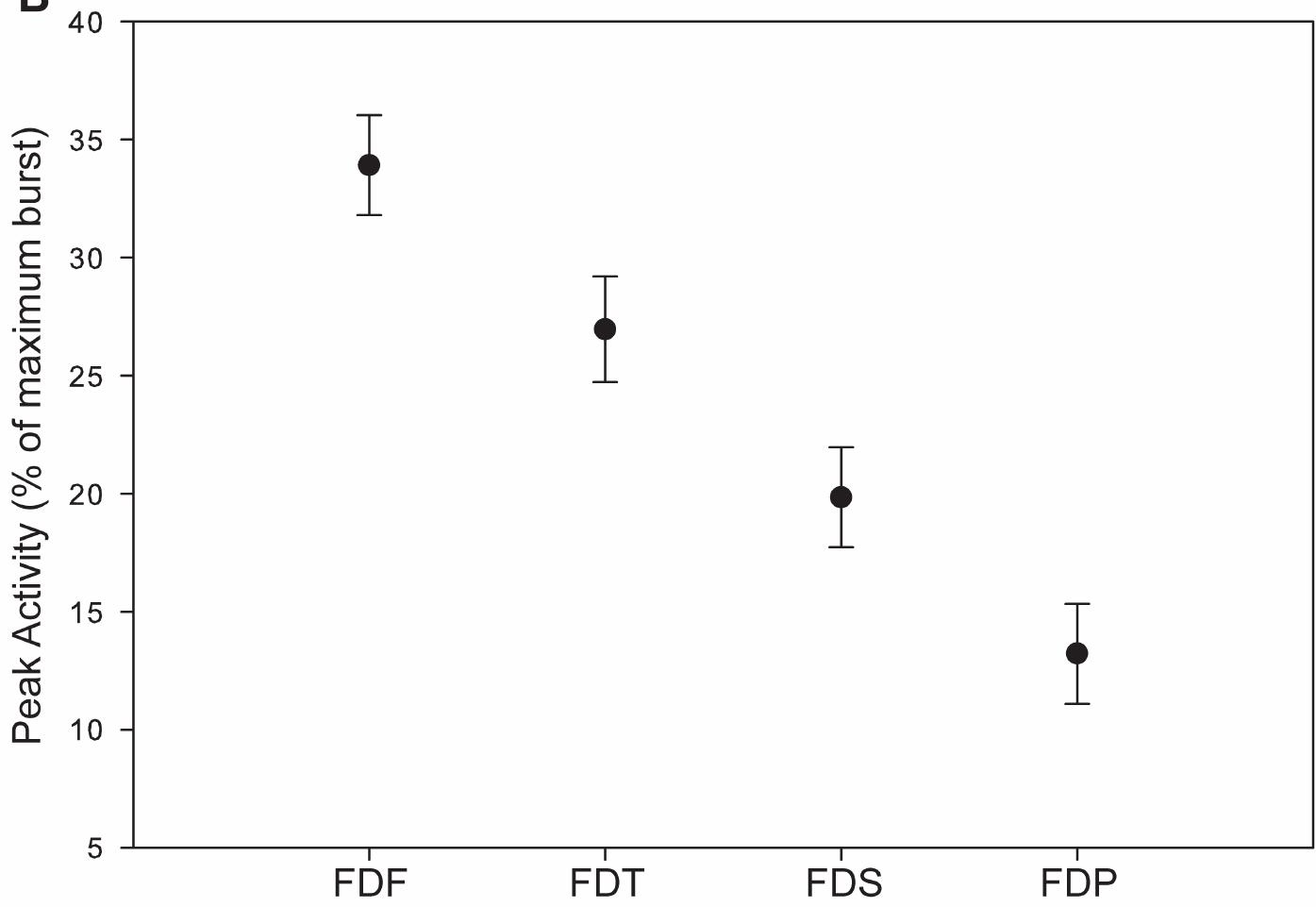


Table 1.

\begin{tabular}{cccccc}
\hline Subject & Experiment \# & FDF & FDT & FDS & FDF \\
A & 1 & 12 & 12 & 13 & 13 \\
A & 2 & 11 & 0 & 9 & 9 \\
B & 1 & 5 & 5 & 5 & 5 \\
C & 1 & 52 & 52 & 53 & 53 \\
& Total Steps: & 80 & 69 & 80 & 80 \\
\hline
\end{tabular}

2

3

$4 \quad$ Table 2.

\begin{tabular}{|c|c|c|c|c|c|}
\hline Variable & Statistic & FDF & FDT & FDS & FDP \\
\hline \multirow[t]{3}{*}{ Relative Duration $^{\dagger}$} & Mean & 89.25 & 65.91 & 53.93 & 28.15 \\
\hline & LS mean & 92.05 & 70.42 & 56.94 & 31.16 \\
\hline & 1 Std. error & 4.756 & 4.765 & 4.765 & 4.899 \\
\hline \multirow[t]{3}{*}{ Relative Peak Activity ${ }^{*}$} & Mean & 35.21 & 28.07 & 21.09 & 14.46 \\
\hline & LS mean & 33.91 & 26.95 & 19.84 & 13.21 \\
\hline & $1 \mathrm{Std}$. error & 2.106 & 2.229 & 2.114 & 2.114 \\
\hline
\end{tabular}

$5+\%$ of stance phase

$6 *$ \% of maximum burst activity

7 